Pacific Journal of Mathematics

ONE SIDED PRIME IDEALS 


\title{
ONE SIDED PRIME IDEALS
}

\author{
JOHN DAUNS
}

Consider a right ideal $L$ in a ring (with $1 \in R$ or $1 € R$ ), its idealizer $N=\{n \in R \mid n L \subseteq L\}$, the bound $P=\{r \in L \mid R r \subseteq$ $L\} \triangleleft R$ of $L$, and the ideal $H=\{n \in N \mid n L \subseteq P\} \triangleleft N$. II. Some of the ideal structure of the ring $N / P$ is determined for a class of one sided prime ideals $L$ more general than the almost maximal ones and without any chain conditions on $R$ (Theorem II). III. When $L \neq P$ the following conditions are necessary and sufficient for $N / P$ to have precisely two unequal, nonzero minimal prime ideals $L / P$ and $H / P$ :

(i) $H \neq P$;

(ii) $L / P<R / P$ is not essential;

(iii) $L / P$ is a maximal annihilator in $R / P$;

(iv) the left annihilator of $L / P$ is not zero;

(v) $L=\{r \in R \mid u r \in P\}$ for some $u \in N \backslash L$ (Theorem III).

Much of the theory of primitive rings arising from a regular maximal right ideal has been generalized to an almost maximal right ideal $L$ in a ring $R$ ([6], [7], and [8]). Thus if $P \subseteq L$ is the biggest ideal of $R$ inside $L$, then $R / P$ is called almost primitive. Recently, the Krull-dimension of modules has received some attention ([9], [11], and [12]), and has also been considered for ordinals rather than integers ([5]). In order to show how the results of [4] are a special case of the present development, the above two apparently distinct conceptsalmost maximal one sided ideals and the Krull-dimension-are related in Theorem I. Some of the ideal structure of the rings $R / P$ and $N / P$ for $L$ almost maximal can be obtained as a special case of a more general result (Theorem II). The latter shows that either $(0)$ is the unique minimal prime ideal of $N / P$, or it has two distinct minimal primes $0 \neq H / P$ and $0 \neq L / P$. If $L \neq P$ and $\boldsymbol{L}<\boldsymbol{R}$ is not essential, then the last alternative holds. However, necessary and sufficient conditions for the latter to hold have to be phrased in terms of the quotient ring $R / P$ (Theorem III). Finally, to see whether some of the results are best possible, some examples and counterexamples are constructed. However, they fail to show that Theorem II is best possible, and this still remains an open question.

1. Preliminaries. Various types of modules and one sided prime ideals are defined.

Notation 1.1. For any ring $\boldsymbol{R}$, define $\boldsymbol{R}^{\mathbf{1}}$ as $\boldsymbol{R}^{\mathbf{1}}=\boldsymbol{R}$ in case $R$ has an identity; otherwise, $\boldsymbol{R}^{\mathbf{1}}=\boldsymbol{Z} \times \boldsymbol{R}$ is the ring with an identity 
adjoined in the usual way, where $Z$ are the integers. Identify $\boldsymbol{R}=$ $\{0\} \times \boldsymbol{R} \triangleleft \boldsymbol{R}^{1}$, where " $\triangleleft$ " will denote two sided while " $<$ " will always denote right ideals. For any right $\boldsymbol{R}$-modules $V$ and $W$, abbreviate $\operatorname{Hom}_{\boldsymbol{R}}(V, W)=(V, W)$. For $B<\boldsymbol{R}$ and $a \in \boldsymbol{R}$, set $\alpha^{-1} B=$ $\{r \in \boldsymbol{R} \mid$ ar $\in B\}<\boldsymbol{R}$.

For $L<\boldsymbol{R}$ a proper right ideal, $\boldsymbol{N}=\{n \in \boldsymbol{R} \mid n L \subseteq L\}$ will denote its idealizer in $\boldsymbol{R}$, while $\boldsymbol{C}$ is the multiplicative semigroup $\boldsymbol{C}=\{x \in$ $\boldsymbol{R} \mid a \in \boldsymbol{R}, x a \in L \Rightarrow a \in L\}$. The term "proper" means $L \neq \boldsymbol{R}$ while $L=$ 0 is permitted. The saturation $\bar{L}$ of any $L<\boldsymbol{R}$ is the right ideal in $L \cong \bar{L}<R$ defined as $\bar{L}=\{x \in R \mid x R \cong L\}$. Note that $(\bar{L})^{-} \neq \bar{L}$ is possible.

Definitions ANd Terminology 1.2. An extension of modules over a ring $\boldsymbol{R}$ is rational if and only if it is rational in the usual sense over $\boldsymbol{R}^{1}$ ([11]). A module has been called strongly uniform $([12 ; 1.4])$ if it is a rational extension of every one of its nonzero submodules. A right ideal $L$ of a ring $\boldsymbol{R}$ is critical ([12; $\S 2]$, or [9; $\S 2]$ and [3]) if the module $\boldsymbol{R} / L$ is strongly uniform.

If $V$ is any right $\boldsymbol{R}$-module, its Krull-dimension-denoted as $k(V)$ is zero if $V$ is Artinian and $V \neq 0$. If $V=0, k(V) \equiv-1$. Let $\beta$ be an ordinal and assume that $k(V)$ has already been defined for all ordinals $\alpha<\beta$ for all modules $V$. Then $k(V)=\beta$ if for every properly descending chain of submodules $V \supset \cdots A \supset B \supset \cdots$, there are at most a finite number of quotients $A / B$ with $k(A / B) \nless \beta$. (I.e., $k(A / B) \nless \beta$ means that it is not the case that either $k(A / B)=\alpha$ for some $\alpha<\beta$ or $k(A / B)$ is undefined.) (See [5; $\S 1]$, or for the case of integers $[4 ; 3.23],[9 ; \S 4]$, or $[10 ; \S 2]$.$) It should be noted that if$ $k(V)$ exists, then $V$ has finite uniform dimension, i.e. $V$ does not contain an infinite direct sum of nonzero submodules ([5; 1.4]).

Any module $V$ is critical, or more precisely, $\alpha$-critical $([5 ; \S 3])$ if $k(V)=\alpha$, but for any submodule $0 \neq W \subset V, k(V / W)<\alpha$. If $\alpha$ is an integer, $k$ will be written in place of $\alpha$.

A module $V$ is compressible provided every nonzero submodule of $V$ contains an isomorphic copy of $V$. By abuse of terminology, sometimes a right ideal $L<R$ is called $\alpha$-critical or compressible provided $\boldsymbol{R} / L$ is.

1.3. A proper right ideal $L<\boldsymbol{R}$ is prime (semi-prime) if for any $s, t \in \boldsymbol{R}$, the inclusion $s \boldsymbol{R}^{1} t \subseteq L\left(s \boldsymbol{R}^{1} s \leqq L\right)$ implies $s \in L$ or $t \in L(s \in L)$.

1.4. A proper $L<\boldsymbol{R}$ is $\Gamma$-prim with respect to a multiplicative subsemigroup $\Gamma \subseteq C$, if 
If $L$ is $C$-prim, then $L$ has already been called (by J. Lambek) prim.

One of the reasons why the next two kinds of prim right ideals are of interest is because there has been a considerable amount of literature about them ([6], [7], and [8]).

Definition 1.5. A right ideal $L<\boldsymbol{R}$ is idealizer complement prim (or $N \backslash L$-prim) if

(1) $N \backslash L \neq \varnothing$ is a multiplicative semigroup;

(2) $\forall s \in R \backslash L, \exists s^{*} \in \boldsymbol{R}$ such that $s s^{*} \in N \backslash L$.

Furthermore, $L<\boldsymbol{R}$ is almost maximal ([7; 5.11] or [8]), if in addition to (1) and (2), the following condition holds

(3) $\forall B<R$ with $L \subset B, L \neq B$ and $\forall a \in R$ :

$$
L \subseteq a^{-2} B \Rightarrow L \subset a^{-} B, L \neq a^{-1} B
$$

2. Compressible and $\alpha$-critical right ideals. Some of the basic facts are summarized below. An identity is not assumed.

2.1. An $\alpha$-critical module is strongly uniform, but not vice versa ([9; 4.2 and 4.3]).

2.2. A nonzero submodule of a strongly uniform (or $\alpha$-critical) module is strongly uniform (or $\alpha$-critical) ([5; 3.5], and [12;1.4] (or $[4 ; 3.23]))$.

2.3. A compressible module with Krull-dimension $\alpha$ is $\alpha$-critical for some $\alpha([5 ; 3.37])$.

2.4. The endomorphism ring of a strongly uniform module is an integral domain. (The proof follows from [12; 2.8]; for rings with identity, see $[12 ; 7.5]$ and $[9 ; 2.9(\mathrm{a})]$.

2.5. For any $L<\boldsymbol{R}, \boldsymbol{N} / \bar{L} \leqq(\boldsymbol{R} / L, \boldsymbol{R} / L)=(\boldsymbol{R} / \bar{L}, \boldsymbol{R} / \bar{L})$. If $L$ is regular, the last inclusion is an equality. Thus if $R / L$ is strongly uniform, then $N / \bar{L}$ is an integral domain. Note that $\bar{L}=L$ if $L$ is semi-prime, and in particular, if almost maximal.

2.6. If $L<R$ is $N \backslash L$-prim (i.e., 1.5(1) and (2) hold), then

(i) $\forall n \in N \backslash L, n(R \backslash L) \subseteq R \backslash L$.

Thus (i) in addition to $1.5(1)$ and (2) implies that

(ii) $\boldsymbol{R} / L$ is compressible.

2.7. If $L<\boldsymbol{R}$ is almost maximal, then

(i) $\boldsymbol{R} / L$ is strongly uniform and 
(ii) $N / L$ is an integral domain with the right Ore condition ([8; 3.1]).

Note that the restrictive hypotheses (ii) (a) and (b) below are not serious because they hold automatically if $\boldsymbol{R}$ has an identity.

For the case when $1 \in \boldsymbol{R}, H$. Storrer ([11]) defines $L<\boldsymbol{R}$ to be almost maximal if and only if $\boldsymbol{R} / L$ is quasi-simple. If $1 \in \boldsymbol{R}$, then the next theorem is an immediate consequence of Storrer's result [11; 7.6]. However, if $1 \notin \boldsymbol{R}$, the above definition of almost maximal is not acceptable and the apparently totally different definition 1.5 has to be used. In $[6 ; 1.3], K$. Koh shows that $R / L$ may be quasisimple without $L$ being almost maximal.

Theorem I 2.8. For a right ideal $L<\boldsymbol{R}$ in any ring $\boldsymbol{R}$, let $\boldsymbol{N}$ be its idealizer and assume that the Krull-dimension of $\boldsymbol{R} / L$ exists. Then the conditions (i) and (ii) are equivalent:

(i) $L$ is an almost maximal right ideal of $\boldsymbol{R}$.

(ii) (a) $L=\bar{L}$ is saturated;

(b) $N \backslash L \neq \varnothing$;

(c) $\boldsymbol{R} / L$ is compressible.

Proof. (i) $\Rightarrow$ (ii). (a) Suppose $x \in \boldsymbol{R} \backslash L$ such that $x \boldsymbol{R} \subseteq L$. Since $x \in N$, by $2.6($ i),$x \boldsymbol{R} \subseteq L$ implies $\boldsymbol{R} \subseteq L$, thus contradicting that $L$ is proper. Thus $L=\bar{L}$. (b) and (c). These conclusions hold by 1.5 and 2.6(ii).

(ii) $\Rightarrow$ (i). By $2.3, \boldsymbol{R} / L$ is $\alpha$-critical, where $\alpha=k(\boldsymbol{R} / L)$. Now 2.1 shows that $R / L$ is strongly uniform. Since $N / L \subseteq(\boldsymbol{R} / L, R / L)$, and since by 2.4 the latter is an integral domain, it follows that (1) $N \backslash L$ is a semigroup. If $s \in \boldsymbol{R} \backslash L$ then $s \boldsymbol{R}+L \neq L$, since $s \boldsymbol{R} \leqq L$ would imply $s \in L$ by hypothesis. There exists a module monomorphism $\psi: R / L \rightarrow(s \boldsymbol{R}+L) / L$. For any $n \in N \backslash L \neq \varnothing$, set $\psi(n+L)=s s^{*}+L \neq L$ where $s^{*} \in R$. The last equation shows that (2) $s s^{*} \in N \backslash L$.

Suppose $L \subset B<\boldsymbol{R}, B \neq L$ and $a \in \boldsymbol{R}$ with $L \subseteq a^{-1} B$ are given as in 1.5 (3). If $a \in L$, then $R=a^{-1} B \neq L$; so let $a \notin L$. Assume $L=$ $a^{-1} B$. But then the module homomorphism $R / L \rightarrow(a \boldsymbol{R}+B) / B$, defined for all $r \in R$ by $r+L \rightarrow a r+B$, is an isomorphism. By assumption, $a \boldsymbol{R} \nsubseteq L$. As was already seen above, $\boldsymbol{R} / L$ is $\alpha$-critical and $\alpha=k(\boldsymbol{R} / L)$. Since $L \subset B, L \neq B$, we have a strict inequality

$$
k(\boldsymbol{R} / B)<\alpha=k(\boldsymbol{R} / L) .
$$

Thus also $k((a \boldsymbol{R}+B) / B)<\alpha$, contradicting that $(a \boldsymbol{R}+B) / B \cong \boldsymbol{R} / L$. Hence the condition (3) that $a^{-1} B \neq L$ holds, and $L$ is almost maximal.

REMARK 2.9. If $\boldsymbol{R}$ is an additive cyclic group of prime order 
with zero multiplication and $L=0$, then $R / L$ is compressible and $k$ critical with $k=0$. However, $L$ is not almost maximal.

A known result is now an immediate corollary of the previous theorem and 2.7(ii).

Corollary to Theorem I 2.10. Consider a right ideal $L<R$ with $L=\{x \in \boldsymbol{R} \mid x \boldsymbol{R} \subseteq L\}$ and proper idealizer $\boldsymbol{N}$, i.e., $\boldsymbol{N} \backslash L \neq \varnothing$. If $\boldsymbol{R} / L$ has a Krull-dimension and is also compressible, then the integral domain $N / L$ satisfies the right Ore condition.

3. Idealizer complement prim right ideals. Some of the results established in [4] for a right Noetherian ring $\boldsymbol{R}$ and a right ideal $\boldsymbol{L}<\boldsymbol{R}$, with $\boldsymbol{R} / L$ compressible and $\alpha$-critical, here will be investigated for much more general rings and right ideals.

Nonation 3.1. Consider any ring $\boldsymbol{R}$, a proper right ideal $L<$ $\boldsymbol{R}$, and as before, its idealizer $N$, semigroup $C$, and saturation $\bar{L}$. By the bound of any subring of $\boldsymbol{R}$ will be understood the unique largest two sided ideal of $R$ contained inside that subring. Let $P$ be the bound of $L$. For any $L<\boldsymbol{R}$, set $\boldsymbol{R}^{\mathbf{1}}: L=\left\{r \in \boldsymbol{R} \mid \boldsymbol{R}^{\mathbf{1}} r \leqq L\right\} \triangleleft \boldsymbol{R}^{\mathbf{1}}$ and similarly for $\boldsymbol{R}: L \triangleleft \boldsymbol{R}^{\mathbf{1}}$. Define a subring $H=\{r \in \boldsymbol{R} \mid r L \leqq P\}$ and $I(H)=\{r \in \boldsymbol{R} \mid H r \cong H\}$. The bound of $H$ in $\boldsymbol{R}$ will be abbreviated as bd $(H)$. The left annihilator $l(L)$ of $L$ in $\boldsymbol{R}$ is $l(L)=\{r \in \boldsymbol{R} \mid r L=$ $0\}$. A right ideal $\{0\} \leqq B \leqq R$ is called an annihilator if there exists a subset $\varnothing \neq S \subseteq \boldsymbol{R}$ such that $B=\{r \in \boldsymbol{R} \mid s r=0$, all $s \in S\}$. Here, for a maximal annihilator right ideal $B \leqq R$, the cases $B=0$ or $B=\boldsymbol{R}$ are also allowed.

The proof of most of the following observations is straightforward and is omitted.

3.2. Without any assumptions on the proper right ideal $L<\boldsymbol{R}$, the following hold:

(1) (i) $R H \subseteq H \subseteq N \leqq I(H), H N \subseteq H$.

(ii) $(H \cap L)^{2} \subseteq H L \subseteq P \subseteq H$; consequently every prime right ideal of $N / P$ contains one of the ideals $H / P \triangleleft N / P$ or $L / P \triangleleft N / P$.

(iii) $\operatorname{bd}(H)=\{x \in \boldsymbol{R} \mid x(\boldsymbol{R} L+L) \subseteq P\}$.

(iv) $P=(\boldsymbol{R}: L) \cap L=\boldsymbol{R}^{1}: L \subseteq \boldsymbol{R}: L$. (The inclusion may be proper. In a ring with zero multiplication, $P=L \subset R: L=R$.)

(v) There is a homomorphism of rings $H \rightarrow(\boldsymbol{R} / L, \boldsymbol{R} / P)$ with kernel $H \cap \bar{P}$.

(2) (i) $H=R \Leftrightarrow N=R \Leftrightarrow L \triangleleft R$, in which case $R=N=H \supset$ $L=P$.

(ii) $H \neq L$, for if $H=L$, then by (1)(i) $R H=R L \leqq H=L$. 
Hence by (2)(i) $H=R=L$, a contradiction since $L$ is proper.

(3) If $L<R$ is regular (in (3) only), i.e., there exists a $u \in R$ with $u r-r \in L$ for all $r \in R$, then

(i) $u \in(N \backslash L) \cap I(H)$; in particular $N \neq L$.

(ii) $P=R: L \leqq L$.

(4) If $L<R$ is semi-prime then

(i) $P=R: L \subseteq L=\bar{L}$,

(ii) and $R: L \triangleleft \boldsymbol{R}$ is semi-prime (see 3.3).

(iii) Furthermore, $L<\boldsymbol{R}$ prime $\Rightarrow P=\boldsymbol{R}: L \triangleleft \boldsymbol{R}$ is also prime (see 3.3).

(iv) $H=N \Leftrightarrow R=N=H \supset L=P$. For if $H=N$, then $L \leqq H$, and hence $L^{2} \subseteq H L \subseteq P$. Thus $L R L \subseteq P$, and $L \subseteq P$.

The next proposition for the prime case is proved by A. W. Goldie in [4] for a right Noetherian ring and a compressible and $k$-critical right ideal. In 3.3-3.5, his results are either used or modified to obtain a theorem applicable to a much wider class of rings that include in particular the rings of most interest here-the almost primitive ones. For a counterexample that 3.3(i) cannot be improved by adding that also $L \triangleleft N$ is prime, see 4.2 and 4.3, where $L>R$ is prime but $L \triangleleft N$ is not.

Proposition 3.3. If $L<\boldsymbol{R}$ is a semi-prime (or respectively, prime) right ideal in any ring $R$ and if $P \subseteq H \subseteq N$ and $b d(H)$ are as above, then

(i) $P \triangleleft \boldsymbol{R}$ and $H \triangleleft N$ are semi-prime (or respectively prime) ideals;

(ii) $N \neq \boldsymbol{R}$ and $H \neq P \Rightarrow H \nsubseteq L$ and $L \nsubseteq H$;

(iii) $L \neq P, L<R$ prime $\Rightarrow b d(H)=P$.

Proof. Note that 3.2(3)(iv) may be used to restate 3.3(ii) as " $H \neq N$ and $H \neq P \Rightarrow \cdots$.. For the prime case, the proof given in [4; 3.42] is applicable and requires only that $R / P$ be prime.

(i) If $s \in \boldsymbol{R}$ with $s \boldsymbol{R}^{\mathbf{1}} s \subseteq P$, then $\left(\boldsymbol{R}^{\mathbf{1}} s\right) \boldsymbol{R}^{\mathbf{1}}\left(\boldsymbol{R}^{\mathbf{1}} s\right) \subseteq L$, hence $\boldsymbol{R}^{1} s \subseteq$ $L$, and $s \in P$.

If $a N^{\mathbf{1}} a \leqq H$ for $a \in N$, then $R^{\mathbf{1}}\left(a L R^{\mathbf{1}} a\right) L \subseteq R^{1}(H) L \subseteq P$. Since $P$ is semi-prime, $R^{\mathbf{1}} a L \subseteq P$. Hence $a L \leqq P$ and $a \in H$.

(ii) If $H \subseteq L$, then $\boldsymbol{R}^{1} H R^{1} \subseteq L$; hence $H R^{1} \subseteq P, H \subseteq P$, and thus $H=P$, which is a contradiction. If $L \subset H$, then $L \boldsymbol{R}^{1} L \subseteq P$. By (i), $L=P$. Hence $N=R$, a contradiction.

(iii) For any $J \triangleleft R$ with $J \subseteq H$, also $J R^{1} L \subseteq P$. Since $L \nsubseteq P$, we have $J \subseteq P$. Thus bd $(H)=P$.

In the next corollary, the proof in $[4 ; 3.42]$ has to be slightly modified by utilizing a result of $[9 ; 2.7]$, because in the corollary $R / L$ is not critical but merely strongly uniform.

COROLlaRY 3.4. With the same notation as in the previous 
proposition, if $L<\boldsymbol{R}$ is a prime right ideal with proper idealizer $\boldsymbol{N} \neq L$ and $\boldsymbol{R} / L$ is strongly uniform, then

(iv) $N \neq R$ and $H \neq P \Rightarrow H / P \triangleleft N / P$ and $L / P \triangleleft N / P$ are distinct minimal prime ideals;

(v) $I(H)=N$.

Proof. (iv) If $L<R$ is prime, $L=\bar{L}$ and 2.5 shows that $N / L$ is an integral domain. By hypothesis $L \neq N$, and thus $L / P \triangleleft N / P$ is proper and hence prime. The relation $(H / P)(L / P)=0$ shows that these are minimal primes.

(v) Clearly $N \subseteq I(H)$. If $x \in I(H)$ and $r \in H \backslash L$ are any elements, then $H x L \subseteq P$ and $r x L \subseteq P \subseteq L$. Here 3.2(2)(ii) and 4(i) have been used. Thus $L+x L \leqq r^{-1} L$ with $r \notin L$. If $L=r^{-1} L$, then $x L \leqq L$. In this case $x \in N$ and hence $I(H)=N$. So assume $L \subset r^{-1} L$ is proper. Any module $R / L$ is strongly uniform if and only if for any right ideal $L \subset B<R$ with $L \neq B,(\boldsymbol{R} / B, E(\boldsymbol{R} / L))=0 \quad([9 ; 2.6]$ and $[11 ; 1.1])$, where $E(\boldsymbol{R} / L)$ is the injective hull of $\boldsymbol{R} / L$. Since $L=\bar{L}, r^{-1} L \neq \boldsymbol{R}$, and

$$
0 \neq \boldsymbol{R} / r^{-1} L \cong(r \boldsymbol{R}+L) / L \cong E(\boldsymbol{R} / L)
$$

contradicts the fact that $\left(\boldsymbol{R} / r^{-1} L, E(\boldsymbol{R} / L)\right)=0$. Thus $I(H)=N$.

In [4], Goldie proves the next theorem for $R / L$ compressible and of Krull-dimension $\alpha$. But then $R / L$ is $\alpha$-critical ([5; 4.6] or [4;3.37]), and by Theorem $I$, then $L$ is almost maximal. However, not every almost maximal right ideal need be of this special latter type. The next theorem is not only applicable to almost maximal right ideals, but even to more general one sided primes.

THEOREM II 3.5. Suppose that in any ring $\boldsymbol{R}$ (with or without identity) the right ideal $L<\boldsymbol{R}$ is idealizer complement prim (1.5). If $P \subseteq L \subset N, P \subseteq H \subseteq N$, and $H \subseteq I(H)$ are as in 3.1, then

(1) $P \triangleleft R, H / P \neq L / P \triangleleft N / P$ are distinct prime ideals.

(2) (a) Either $H=P$, or $H=N$ and $H / P, L / P \triangleleft N / P$ are two distinct prime ideals, one of which is always zero, ordered by inclusion as follows

$$
\begin{aligned}
& H=P \Longleftrightarrow H \subset L, \\
& H=N \Longleftrightarrow L \subset H \Longleftrightarrow H=N=R \supset L=P ;
\end{aligned}
$$

(b) or $H \neq P$ and $H \neq N$ (in which case $L \neq P$ ) and the ring $N / P$ has exactly two distinct minimal nonzero prime ideals $H / P \neq L / P \triangleleft N / P$.

(3) The bound of $H$ in $\boldsymbol{R}$ is $P$.

(4) $I(H)=N$.

Proof. First (without any assumptions), by 3.2(2)(ii), $H \neq L$. 
Hence $H / P \neq L / P$ are always distinct.

(1) By 3.3(i) and 1.5(1) the ideals $H / P, L / P \triangleleft N / P$ are always prime.

(2) (a) If $H \subset L$, then $\boldsymbol{R}^{1} H \subseteq H \subseteq L$, hence $H \subseteq P$, and $H=P$.

If either $L \subset H$, or if $H=N$, in both cases we have $L \subset H$. But then $L^{2} \subseteq H L \subseteq P$ and $P \triangleleft \boldsymbol{R}$ is prime. Hence $L=P \subset \boldsymbol{R}=$ $N=H$.

(b) By (2)(a), $H \nsubseteq L, L \nsubseteq H, L \neq P$ and $N \neq R$. (Alternative proof: By 3.3(ii), $H \nsubseteq L$ and $L \nsubseteq H$, i.e., $H / P$ and $L / P$ are not ordered by inclusion.) Finally by $3.2(1)(i i),(H / P)(L / P)=0$ shows that every prime ideal of $N / P$ contains either $H / P$ or $L / P$. The latter together with 3.5(1) shows that these are minimal primes and that there are no others in $N / P$.

(3) is a consequence of 3.3 .

(4) If as before $x \in I(H)$ and $r \in H \backslash L$, then $r x L \subseteq P$ and $L+$ $x L \cong r^{-1} L$. Suppose $x L \nsubseteq L$. Let $a \in L$ with $x a \notin L$. By 1.5(2), choose $t \in \boldsymbol{R}$ such that $(x a) t \in N \backslash L$. But then $r \in H \backslash L \subseteq N \backslash L, x a t \in N \backslash L$, while $r(x a t) \in r x L \subseteq L$ contradicts that $N \backslash L$ is a semigroup.

It remains yet to determine necessary and sufficient conditions for each of the two alternatives 2(a) or (b) in Theorem II to hold. The next lemma and proposition not only establish, clarify, and supplement such necessary and sufficient conditions, but are also of independent interest.

Lemma 3.6. An idealizer complement prime right ideal $L<\boldsymbol{R}$ and its left annihilator $l(L)$ in $\boldsymbol{R}$ satisfy the following:

(i) $\forall u \in l(L) \backslash L \Rightarrow L=u^{-1} 0$.

(ii) $L<\boldsymbol{R}$ is not essential $\Leftrightarrow l(L) \backslash L \neq \varnothing$.

(iii) If in addition $\boldsymbol{R}$ is semi-prime, then there does not exist $B \leqq R$ with $L \subset B, L \neq B$, and $l(B) \neq 0$.

Proof. (i) Since $l(L) \backslash L \subseteq N \backslash L$, and since $n(\boldsymbol{R} \backslash L) \leqq \boldsymbol{R} \backslash L$ for all $n \in N \backslash L$, it follows that $L=u^{-1} 0$.

(ii) $\Rightarrow$ : There is a right ideal $0 \neq K \leqq R$ with $K \cap L=0$. Hence $K \cap(N \backslash L) \neq \varnothing$. Since $(K \cap N) L \subseteq K \cap L=0$, it follows that $K \cap$ $(N \backslash L) \subseteq l(L) \backslash L \neq \varnothing$.

(ii) $\leftleftarrows:$ For any $n \in N \backslash L, r \in R, n r \in L$ implies that $r \in L$. Thus $n \boldsymbol{R} \cap L=n L$. But $l(L) \backslash L \leqq N \backslash L$. Thus for any $u \in l(L) \backslash L, u \boldsymbol{R} \neq 0$ and $u \boldsymbol{R} \cap L=u L=0$.

(iii) Any right ideal $B \leqq \boldsymbol{R}$ in a semi-prime ring $\boldsymbol{R}$ with $l(B) \neq$ 0 is proper and satisfies $l(B) \backslash B \neq \varnothing$. For suppose $l(B) \subseteq B$. Then $l(B) R^{1} l(B) \subseteq l(B) B=0$ and hence $l(B)=0$. Suppose that there does exist a $B \leqq \boldsymbol{R}$ as in (iii). (E.g., an annihilator right ideal $B$ properly 
containing $L$.) Since $L \subset B$ we have $l(B) \backslash B \subseteq l(L) \backslash L \subseteq N \backslash L$. Take any $x \in l(B) \backslash B$ and any $b \in B \cap(N \backslash L)$. Then $x b=0$ with $x, b \in N \backslash L$ contradicts that $N \backslash L$ is a semigroup.

For almost maximal right ideals, conclusion (ii) of the next proposition is due to K. Koh (verbal communication, also see [8; 2.3]).

Proposition 3.7. If $L<\boldsymbol{R}$ is an idealizer complement prime right ideal in any ring $\boldsymbol{R}(1 \in \boldsymbol{R}$ or $1 \notin \boldsymbol{R})$, then.

(i) $L<R$ is not essential $\Leftrightarrow L=u^{-1} 0$ for some $u \in R \backslash L$.

If in addition it is assumed that $\boldsymbol{R}$ is semi-prime, then

(ii) $L<\boldsymbol{R}$ is not essential $\Leftrightarrow L$ is a maximal annihilator.

Proof. (i) follows from 3.6(ii) and (i).

(ii) $\Rightarrow$ : Again by 3.6 (ii), or alternatively by $3.7(\mathrm{i}), L$ is an annihilator. The semi-primeness of $\boldsymbol{R}$ together with 3.6(iii) shows that $L$ is a maximal annihilator.

(ii) $\Longleftarrow$ : Since $l(L) \neq 0$ and $R$ is semi-prime the argument used in the proof of 3.6(iii) shows that $l(L) \backslash L \neq \varnothing$. But now 3.6(ii) shows that $L<\boldsymbol{R}$ is not essential.

Since the case $H=R$ is uninteresting, the next theorem determines exactly when each of the two alternatives in Theorem II hold.

THeOREM III 3.8. Suppose that $L<\boldsymbol{R}$ is idealizer complement prim (1.5) in any ring $\boldsymbol{R}(1 \in \boldsymbol{R}$ or $1 \notin \boldsymbol{R})$, and that $H, P$ and $l(L / P)$ are as previously (3.1). The following conditions are all equivalent:

(i) $H \neq P$.

(ii) $L / P<R / P$ is not essential.

(iii) $L / P$ is a maximal annihilator in $R / P$.

(iv) $l(L / P) \backslash L / P \neq \varnothing$.

(v) $L=u^{-1} P$ for some $u \in R \backslash L$.

Proof. Since $P \triangleleft \boldsymbol{R}$ is prime by 3.3(i), the equivalence of (ii)(v) follows from 3.6 and 3.7. Only in this proof and no where else is the notation $\bar{L}$ other than the saturation of $L$. Set $\bar{L}=L / P<\overline{\boldsymbol{R}}=$ $\boldsymbol{R} / P, \bar{P}=0$, and $\bar{H}=H / P \bar{N}=N / P$. Since the idealizer of $\bar{L}$ in $\overline{\boldsymbol{R}}$ is $\bar{N}$, also $\bar{L}<\overline{\boldsymbol{R}}$ is idealizer complement prim. However, $l(\bar{L})=\bar{H}$. The " $H$ associated with any right ideal $L$ by 3.1 " for $\bar{L}$ is $\bar{H}$, i.e., $\{\bar{x} \in$ $\bar{R} \mid \bar{x} \bar{L} \subseteq \bar{P}\}=H / P=l(\bar{L})$. Application of 3.6 (ii) to $\bar{L}<\bar{R}$ in place of $L<R$ shows that

$$
\bar{L}<\bar{R} \text { is not essential } \Longleftrightarrow l(\bar{L}) \backslash \bar{L} \neq \varnothing \Longleftrightarrow \bar{H} \backslash \bar{L} \neq \varnothing .
$$

More generally, without any special assumptions on $L<\boldsymbol{R}$ whatever

$$
H \backslash P \neq \varnothing \circlearrowright H \backslash L \neq \varnothing \Longleftarrow \bar{H} \backslash \bar{L} \neq \varnothing \Longleftarrow \bar{H} \neq 0 .
$$


For if $h \in H \backslash P$, then $h L \subseteq P=R^{1}: L$, while $R^{1} h \nsubseteq L$. Thus $\rho h \notin L$ for some $\rho \in \boldsymbol{R}^{1}$ but $\rho h \in \boldsymbol{R}^{1} H \backslash L=H \backslash L$. Hence $\bar{L}<\bar{R}$ is not essential iff $H \neq P$.

For a right Noetherian ring the next corollary is stated in [4; 3.42]. It is a special case of 3.8. The converse implication below fails for maximal ideals $L$ in a commutative semi-prime ring $\boldsymbol{R}$ with $\boldsymbol{R}=H \neq P=L$, where $L$ and $\boldsymbol{R}$ can be so chosen that $L<\boldsymbol{R}$ either is, or is not essential.

CoRollary to TheOREM III 3.9. With the same hypotheses and notation as in the last theorem

$$
L<\boldsymbol{R} \text { is not essential } \Rightarrow H \neq P \text {. }
$$

CoRollary to Theorems II AND III 3.10. With the hypotheses and notation of Theorem II,

(2) (a) $H=P \Leftrightarrow L / P<R / P$ is essential.

(a) $H=N \Leftrightarrow L=P$.

(b) $H \neq N, H \neq P \Leftrightarrow L \neq P$, and $L / P<R / P$ is not essential.

4. Examples. It has to be determined by means of counterexamples how much of Theorem II and its Corollaries could still be proved under the following possibilities for weakening the hypotheses on the right ideal $L<R$ :

(1)(a) prim irreducible, (b) prim; (2)(a) prime irreducible, or (b) prime, where $L<R$ is irreducible if it is not the intersection of two right ideals properly bigger than $L$.

4.1. Throughout, $F$ will be a field. In the free $\operatorname{ring} \boldsymbol{R}=F[x, y]$ of noncommuting polynomials in indeterminates $x$ and $y$, the right ideal $L=x \boldsymbol{R}<\boldsymbol{R}$ is prim irreducible, but not $N \backslash L=F-$ prim. Conclusion (4) of Theorem II fails, for $H=0$ and $I(H)=\boldsymbol{R}$, but not $N$ as required by (4). The Corollary 3.9 to Theorem II also fails, for $L<\boldsymbol{R}$ is not essential while $H=P=0$.

Next a prim right ideal $L<\boldsymbol{R}$ will be constructed such that $L<N$ is not prime in its idealizer.

4.2. Suppose $\sigma: F \rightarrow F$ is a one-to-one homomorphism of $F$ into $F$ but not onto. Set $E=(F \sigma) \sigma \subset F \sigma \neq F$. Consider the twisted polynomial ring $\boldsymbol{R}=F[x, \sigma]$ with coefficients on the left and $x^{n} c=$ $c \sigma(n) x^{n}$ for $c \in F$ and $\sigma(n) \equiv \sigma^{n}$ for $0 \leqq n \in Z$, where $Z$ is the ring of integers. Then $R$ is a left but not right Ore domain. View elements $\alpha, \beta \in \boldsymbol{R}$ as functions $a, \beta: Z \rightarrow \boldsymbol{R}$ that are eventually zero and vanish on the negative integers under pointwise addition and the multiplication 
$(\alpha \beta)(n)=\Sigma\{\alpha(i)[\beta(j) \sigma(i)] \mid i, j \in Z ; i+j=n\}$ for $n \in Z$. Denote by $E[x$, $\sigma] \subset R$ the subring of all polynomials with left coefficients in $E$, i.e., $E[x, \sigma]=\{\alpha \in \boldsymbol{R} \mid \alpha(\boldsymbol{Z}) \subseteq E\}$.

4.3. The right ideal $L=x^{2} R<R$ is also given by $L=E[x, \sigma] x^{2}$. Its idealizer $N$ is $N=E[x, \sigma]$. Since $x N x \subseteq L$ with $x \in N \backslash L, L$ when viewed as an ideal in the subring $L \triangleleft N$ is not even semi-prime; consequently, conclusion (1) of Theorem II is false. It should also be noted that $H=0=P, I(H)=\boldsymbol{R}$. Consequently, (4) of Theorem II fails again.

In order to see that $L$ is reducible, take any scalar $f \notin F \sigma$ and form $(f x \boldsymbol{R} \oplus L) \cap(x f x \boldsymbol{R} \oplus L)=L$, where

$$
f x \boldsymbol{R} \cap L=x f x \boldsymbol{R} \cap L=f x \boldsymbol{R} \cap x f x \boldsymbol{R}=0 .
$$

If $\alpha=\alpha(k) x^{k}+\alpha(k+1) x^{h+1}+\cdots, \beta=\beta(m) x^{m}+\cdots \in R$ with $\alpha \boldsymbol{R} \beta \leqq$ $L$, then $\alpha \beta(m)^{-1} \beta \in L$ implies that $\alpha(k) \in E$, while $\alpha f \beta(m)^{-1} \beta \in L$ shows that $k \geqq 2$. Hence $\alpha \in L$. It will be shown below that $L<R$ is actually prim with respect to a subsemigroup $\Gamma \cong C$.

4.4. Define $\Gamma \leqq \boldsymbol{C}$ as the subsemigroup $\Gamma=\{\gamma \in \boldsymbol{C} \mid \gamma(\boldsymbol{R} \backslash\{0\}) \subseteq$ $\boldsymbol{R} \backslash L\}$. First we must determine when a typical element $p=p(m) x^{m}+$ $\cdots+p(u) x^{u} \in \boldsymbol{R}$ belongs to $\Gamma$, where the first and last nonzero coefficients of $p$ are $p(m), p(u) \in F$ with $m \leqq u$. Since $p \in \Gamma$ implies $p+$ $L \subseteq \Gamma$, and since $L=E[x, \sigma] x^{2}$, without loss of generality assume that for $j \geqq 2$, either $p(j) \notin E$ or $p(j)=0$. Throughout, it will be assumed that $p \alpha \in L$ with $\alpha=\alpha(k) x^{k}+\cdots+\alpha(s) x^{s} ; \alpha(k) \neq 0, \alpha(s) \neq 0$, and $k \leqq s$.

Proposition 4.5. With $p \in \boldsymbol{R}$ and $m$ the degree of $p$ satisfying the conventions in 4.4,

(a) $p \in \Gamma$ provided either one of the following two conditions hold:

(1) $m \geqq 2$;

(2) $p=p(1) x+\cdots ; \quad m=1, p(1) \notin F \sigma$.

Furthermore,

(b) $L<\boldsymbol{R}$ is $\Gamma$-prim.

Proof. (a)(1). For $0 \neq \alpha \in \boldsymbol{R}, p \alpha \in L$ implies $p(m)[\alpha(k) \sigma(m)] \in E$, which in turn gives the contradiction that $0 \neq p(m) \in E$ for $m \geqq 2$.

(a)(2) Now $p \alpha \in L$ requires that $k \geqq 1$ and $p(1)[\alpha(k) \sigma] \in E$, which contradicts that $p(1) \notin F \sigma$.

(b) Let $\alpha=\alpha(k) x^{k}+\cdots+\alpha(s) x^{s} \in \boldsymbol{R} \backslash L$ with $k \leqq s$. If $\gamma \in \Gamma$, then $\gamma+L \subseteq \Gamma$. Hence for any $\lambda \in R$, the condition $(\alpha+L) \lambda \cap \Gamma \neq$ $\varnothing$ is equivalent to $\alpha \lambda \in \Gamma$. Consequently, it may be assumed without any loss of generality that for $j \geqq 2$, either $\alpha(j)=0$ or $\alpha(j) \notin E$. Take 
any $f \in F \backslash F \sigma$. Then $\alpha \notin L$ is one of the following four mutually exclusive forms

$$
\begin{array}{llrl}
k \geqq 2 ; & \alpha(k) \notin E & \Longrightarrow & \alpha \in \Gamma \\
k=0 ; & & \Longrightarrow \alpha \alpha(0){ }^{1} f x^{2} \in \Gamma \\
k=1 ; & \alpha(1) \notin F \sigma \Longrightarrow & & \alpha \in \Gamma \\
k & =1 ; & \alpha(1) \in F \sigma . &
\end{array}
$$

In the last and only remaining case $\alpha(1)=c \sigma$ for a unique $c \in F$. Since $f \sigma \notin E$, (a)(1) shows that $\alpha c^{-1} f x^{2}=(f \sigma) x^{3}+\cdots \in \Gamma$. Thus for any $\alpha \in R \backslash L, \alpha F f x^{2} \cap \Gamma \neq \varnothing$ and $L<R$ is $\Gamma$-prim.

REMARK 4.6. With $p \in \boldsymbol{R}$ as in 4.4, $p \in \Gamma$ if any one of the following conditions hold:

(3) $u \geqq 2$ (and hence $p(u) \notin E$ ).

(4) $u=1$ and $p(1) \notin F \sigma$.

Furthermore, $p \in \boldsymbol{C}$ in case any one of the following hold:

(v) $0 \neq p(m) \in E$ and $p(m+i) \notin E$ for some $i \geqq 1$.

(To prove $4.6(\mathrm{v})$, it suffices to take $p=p(0)+p(1) x$ with $0 \neq p(0) \in E$ but $p(1) \notin E$. For if $m+i \geqq 2$, then also $u \geqq 2$ and $4.6(3) \Rightarrow 4.6(\mathrm{v})$. So $m+1 \leqq m+i<2$ and $m=0, i=1$.)

(vi) $p \in E[x, \sigma]$ with $p(0) \neq 0$.

\section{REFERENCES}

1. J. Dauns, Multiplier rings and primitive ideals, Trans. Amer. Math. Soc., 145 (1969), 124-158.

2. Wimple modules and centralizers, Trans. Amer. Math. Soc., 166 (1972), 457-477.

3. C. Faith and Y. Utumi, Quasi-injective modules and their endomorphism rings, Arch. Math., Vol. XV (1964), 166-174.

4. A. W. Goldie, The Structure of Noetherian Rings, Tulane Ring Year Lecture Notes, Lecture Notes in Mathematics No. 246, Springer (1972), New York.

5. R. Gordon and J. C. Robson, Krull-dimension and monoform modules, to appear.

6. K. Koh, On almost maximal right ideals, Proc. Amer. Math. Soc., 25 (1970), 266-272.

7. - Quasi-Simple Modules and Other Topics in Ring Theory, Tulane Ring Year Lecture Notes, Lecture Notes in Mathematics No. 246, Springer (1972), New York.

8. K. Koh and A. Mewborn, A class of prime rings, Canad. Math. Bull., 9 (1966), 6372.

9. J. Lambek and G. Michler, The torsion theory at a prime ideal of a right Noetherian ring, to appear.

10. J. C. Robson, Idealizers and hereditary Noetherian prime rings, J. Algebra, 22 (1972), 45-81.

11. H. Storrer, Rational extensions of modules, Pacific J. Math., 38 (1971), 785-794.

12. - On Goldman's Primary Decomposition, Tulane Ring Year Lecture Notes, Lecture Notes in Mathematics No. 246, Springer (1972), New York.

Received May 8, 1972 and in revised form February 3, 1973. This research was partially supported by a National Science Foundation grant. 


\section{PACIFIC JOURNAL OF MATHEMATICS}

\section{EDITORS}

D. Gilbarg AND J. MILGRAM

Stanford University

Stanford, California 94305

\section{R. A. Beaumont}

University of Washington

Seattle, Washington 98105

\section{J. DUGUNDJI*}

Department of Mathematics University of Southern California Los Angeles, California 90007

RICHARD ARENS

University of California Los Angeles, California 90024

\section{ASSOCIATE EDITORS}
E. F. BECKENBACH
B. H. NEUMANN
F. WOLF
K. YoshidA

\section{SUPPORTING INSTITUTIONS}

\author{
UNIVERSITY OF BRITISH COLUMBIA \\ CALIFORNIA INSTITUTE OF TECHNOLOGY \\ UNIVERSITY OF CALIFORNIA \\ MONTANA STATE UNIVERSITY \\ UNIVERSITY OF NEVADA \\ NEW MEXICO STATE UNIVERSITY \\ OREGON STATE UNIVERSITY \\ UNIVERSITY OF OREGON \\ OSAKA UNIVERSITY
}

\author{
UNIVERSITY OF SOUTHERN CALIFORNIA \\ STANFORD UNIVERSITY \\ UNIVERSITY OF TOKYO \\ UNIVERSITY OF UTAH \\ WASHINGTON STATE UNIVERSITY \\ UNIVERSITY OF WASHINGTON \\ AMERICAN MATHEMATICAL SOCIETY \\ NAVAL WEAPONS CENTER
}

The Supporting Institutions listed above contribute to the cost of publication of this Journal, but they are not owners or publishers and have no responsibility for its content or policies.

Mathematical papers intended for publication in the Pacific Journal of Mathematics should be in typed form or offset-reproduced, (not dittoed), double spaced with large margins. Underline Greek letters in red, German in green, and script in blue. The first paragraph or two must be capable of being used separately as a synopsis of the entire paper. Items of the bibliography should not be cited there unless absolutely necessary, in which case they must be identified by author and Journal, rather than by item number. Manuscripts, in duplicate if possible, may be sent to any one of the four editors. Please classify according to the scheme of Math. Rev. Index to Vol. 39. All other communications to the editors should be addressed to the managing editor, Richard Arens, University of California, Los Angeles, California, 90024.

50 reprints are provided free for each article; additional copies may be obtained at cost in multiples of 50 .

The Pacific Journal of Mathematics is issued monthly as of January 1966. Regular subscription rate: $\$ 48.00$ a year (6 Vols., 12 issues). Special rate: $\$ 24.00$ a year to individual members of supporting institutions.

Subscriptions, orders for back numbers, and changes of address should be sent to Pacific Journal of Mathematics, 103 Highland Boulevard, Berkeley, California, 94708.

PUBLISHED BY PACIFIC JOURNAL OF MATHEMATICS, A NON-PROFIT CORPORATION

Printed at Kokusai Bunken Insatsusha (International Academic Printing Co., Ltd.), 270, 3-chome Totsuka-cho, Shinjuku-ku, Tokyo 160, Japan.

* C. DePrima will replace J. Dugundji until August 1974.

Copyright (C) 1973 by

Pacific Journal of Mathematics

All Rights Reserved 


\section{Pacific Journal of Mathematics}

\section{Vol. 47, No. $2 \quad$ February, 1973}

David Parham Bellamy, Composants of Hausdorff indecomposable continua; a mapping approach ........................ 303

Colin Bennett, A Hausdorff-Young theorem for rearrangement-invariant spaces ...........................................

Roger Daniel Bleier and Paul F. Conrad, The lattice of closed ideals and $a^{*}$-extensions of an abelian l-group ...

Ronald Elroy Bruck, Jr., Nonexpansive projections on subsets of Banach

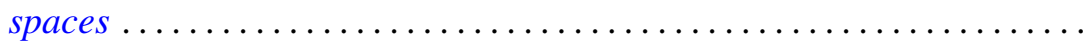

Robert C. Busby, Centralizers of twisted group algebras ............. 357

M. J. Canfell, Dimension theory in zero-set spaces ................ 393

John Dauns, One sided prime ideals ........................ 401

Charles F. Dunkl, Structure hypergroups for measure algebras . . . . . . . . . 413

Ronald Francis Gariepy, Geometric properties of Sobolev mappings ...... 427

Ralph Allen Gellar and Lavon Barry Page, A new look at some familiar spaces of intertwining operators ...........................

Dennis Michael Girard, The behavior of the norm of an automorphism of the

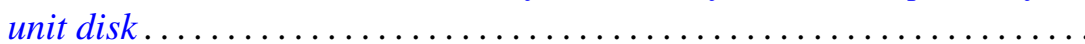

George Rudolph Gordh, Jr., Terminal subcontinua of hereditarily

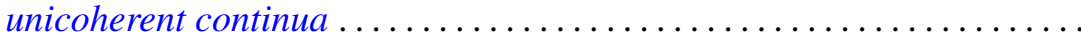

Joe Alston Guthrie, Mapping spaces and cs-networks. .

Neil Hindman, The product of $F$-spaces with $P$-spaces . 473

M. A. Labbé and John Wolfe, Isomorphic classes of the spaces $C_{\sigma}(S)$

Ernest A. Michael, On k-spaces, $k_{R}$-spaces and $k(X) \ldots$

Donald Steven Passman, Primitive group rings .

C. P. L. Rhodes, A note on primary decompositions of a pseudovaluation ...

Muril Lynn Robertson, A class of generalized functional differential equations

Ruth Silverman, Decomposition of plane convex sets. $I$.

Ernest Lester Stitzinger, On saturated formations of solvable Lie algebras................................

B. Andreas Troesch, Sloshing frequencies in a half-space by Kelvin inversion ...

L. E. Ward, Fixed point sets .

Michael John Westwater, Hilbert transforms, and a problem in scattering

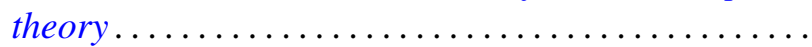

Misha Zafran, On the spectra of multipliers... 